\title{
Numerical simulation of extended structures aerodynamic with high level of responsibility
}

\author{
Anastasia Fedosova ${ }^{1, *}$, Yulia Gribach $^{1}$, and Dmitry Gribach ${ }^{1}$ \\ ${ }^{1}$ Moscow state university of civil engineering, Yaroslavskoye sh. 26, 129337 Moscow, Russia
}

\begin{abstract}
Using numerical simulation the paper investigates the aerodynamics of extended airport complex; according to the classification of existing regulations the complex is related to buildings and structures of high level of responsibility. We present the technique used for numerical simulation and the results obtained by the application of numerical simulation, the results were verified with the results obtained during physical modelling in wind tunnel of architectural type. According to the results of the research the conclusions about the pattern of flow around the building wind flow were made.
\end{abstract}

\section{Introduction}

Airports buildings are classified as buildings and structures of high level responsibility. The majority of these facilities are unique space-planning solutions. Construction of these facilities is a complex engineering task.

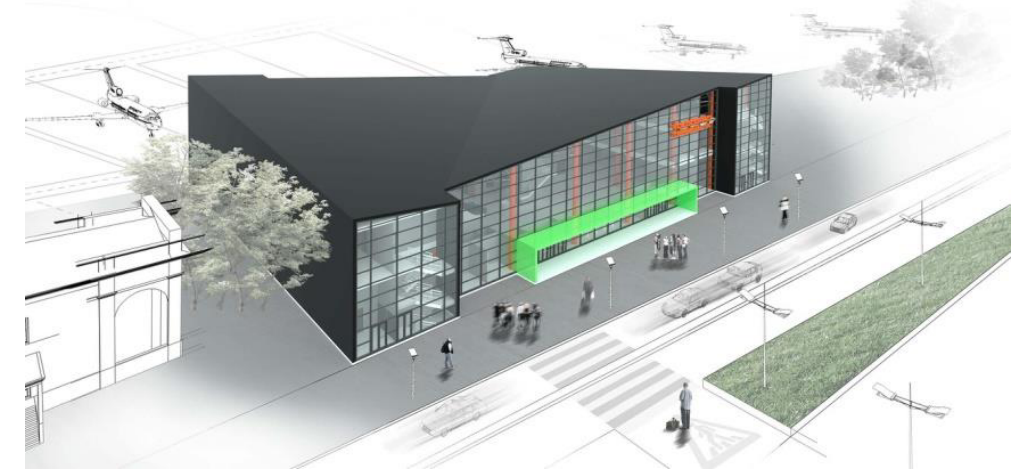

Fig. 1. Extended airport complex.

The considered airport (Fig.1) is a large-span structure in the form of a rectangular with volume terms (axes dimensions are $50 * 70 \mathrm{~m}$ ) and varying elevations. The roof has two ramp from two opposite angles; minimum height is $13.45 \mathrm{~m}$ and a maximum reach is $19 \mathrm{~m}$. The given design features allow to categorize the complex as unique extended structure of buildings and structures of increased level of responsibility. For this class of

* Corresponding author: $\underline{\operatorname{mgsu} @ \text { broll.ru }}$ 
building there is no data on the distribution of wind load and acting domestic and foreign building codes and regulations prescribe wind parameter assignment by the results physical modelling. In this paper a study of wind loads will be produced using the numerical simulation and the verification of the obtained results with the results of physical modelling.

\section{Numerical simulation}

The creation of three-dimensional geometric model is preceded the process of calculating the aerodynamic characteristics of the building. 3D geometric model must correspond to the original configuration of the object. 3D-body was projected on the basis of the analysis of space-planning and constructive solutions to the passenger terminal on the principle of similarity, which, in consequence of different scale transforms unstructured grids (Fig. 2). The grid is defined by the necessary number of refinements (in this case: the number of volumes of more than 5 million).

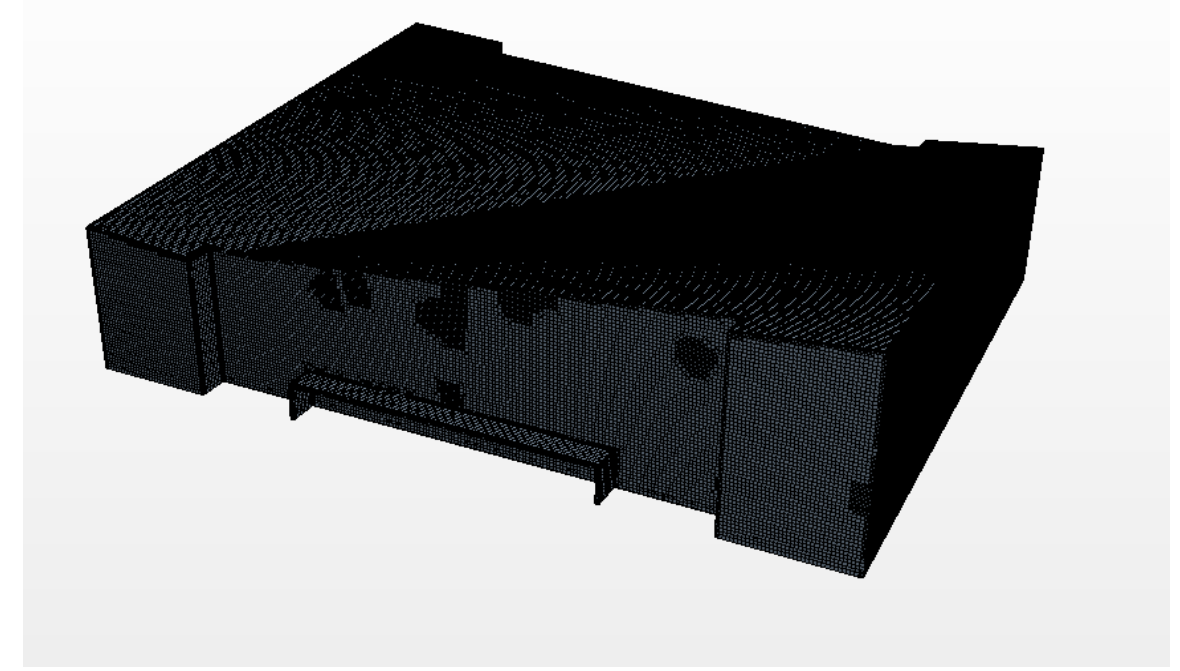

Fig. 2. Detail of the grid near the building.

The next stage of the study is setting the boundary conditions: the entrance of the computational domain (inlet) is defined by the velocity flow $U(z)$, the intensity of turbulent pulsations I and the scale of turbulence; the orientation of the wind direction is defined as well; the condition of constant pressure is set on the output of the calculated area.

- the entrance of the computational domain (inlet) is defined by the velocity flow $U(z)$, the intensity of turbulent pulsations I and the scale of turbulence

- the orientation of the wind direction is defined as its shows at Fig. 3;

- the condition of constant pressure is set on the output of the calculated area;

- on the bottom, on top of the remote and on the internal borders (on the facades of buildings and surrounding objects) - no-slip wall - zero speed (parietal cells height was $2.5 \cdot 10^{-4} H$ that provided $\mathrm{y}+$ about 1 and made it possible not to use the model wall functions).

In this study, for determination the aerodynamic characteristics with a numerical modelling software package Ansys Fluent 14.5 is used. The structure of the performing 
numerical simulation of wind influence has the following order: the three-dimensional configuration of the body flows about the turbulent flow of an incompressible air, having an average distribution rate adjustment calculation domain in accordance with the experimental velocity profile and intensity of turbulent pulsations. As the calculated value a dimensionless pressure coefficient $\mathrm{Cp}$ is used, which is distributed on the facades around the perimeter of the horizontal building section. The turbulent motion of the air environment near the volume described by the system of Reynolds equations.

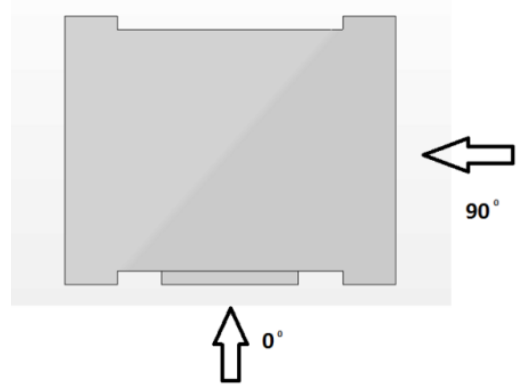

Fig. 3. Directions wind model in numerical simulation (top view).

The essence of this method consists in that all the instantaneous flow values of studied parameters are presented as the sum of the averaged value of the pulse and its component, i.e. the resulting value is random, which in time averaging gives her expectation, and the fluctuating component of which is the variance of the random variable. However, using only the solution of the basic equations makes the system appears to open, and finding the unknown quantity becomes impossible. For solving the system the system closure is made by bringing additional equations that govern the laws of turbulence development.

\section{Verification}

Obtained by computer simulation the distribution of average pressure on the facades of opportunity should be verified with the data that was determined experimentally. For this a number of drainage points on the model and layout was chosen. A series of preliminary calculation was performed for a number of areas, and then a comparative analysis of the results was performed (Fig. 4).

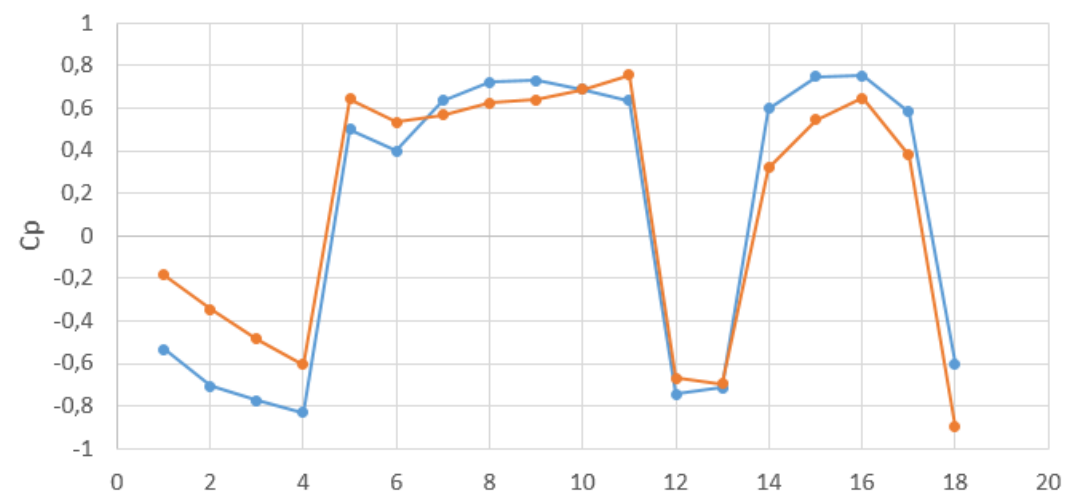

Fig. 4. The distribution of the average coefficient of aerodynamic pressure at verification points along the perimeter of the airport terminal complex with flow angle equal to $0^{\circ}$ (read - experimental, blue numerical). 
Carrying out data verification allows choosing the most rational parameters of the computational grid, turbulence model and its characteristics, providing relatively little difference between the calculated and experimental results. In this paper, it does not exceed $20 \%$.

\section{The results of numerical simulation}

The complex configuration of the airport leads to the formation of numerous vouchers and jet vortex zones, which form a non-monotonic pattern of distribution of wind pressure on the facades of buildings. Fig. 5 gives a picture of the distribution of the streamlines at a height of $15 \mathrm{~m}$ for the flow angles of $0^{\circ}$ and $90^{\circ}$, and in Fig. 6 shows a picture of the distribution average coefficient of aerodynamic pressure on the facades of the airport complex.

a

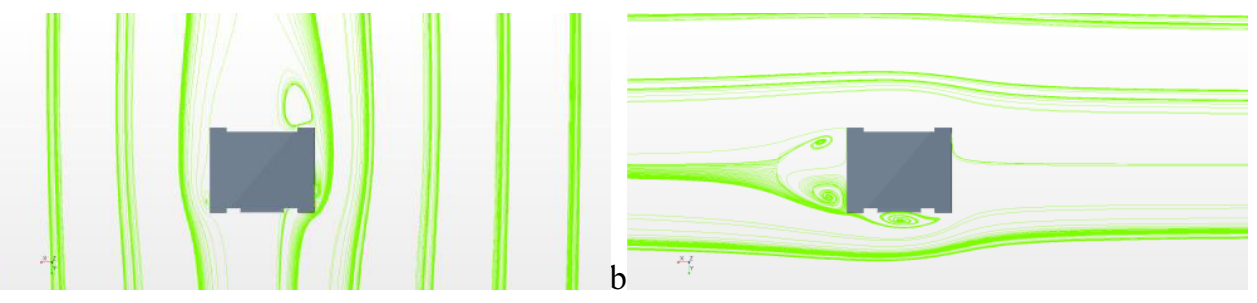

Fig. 5. Streamlines of $15 \mathrm{~m}$ at flow angle $0^{\circ}$ (a) and $90^{\circ}$ (b).

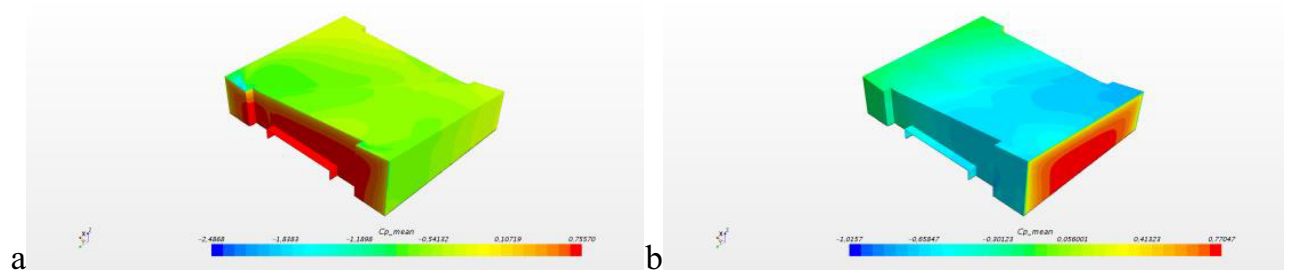

Fig. 6. The distribution of the average aerodynamic pressure on the facades rate of flow at an angle of $0^{\circ}$ (a) and $90^{\circ}(\mathrm{b})$.

Simulation of flow around the building using the computer technology provides information on distributed wind loads on the facade of the object just as it is done by physical experiment in conducting drainage tests of object models in a wind tunnel. To present the results of a "virtual experiment" "drainage point" were selected virtual, which was defined wind pressure coefficient (average component) according to the aerodynamic formula

$$
C_{\text {pmean }}=\frac{w_{m}}{0.5 \rho U^{2}(H)},
$$

where $w_{m}$ is calculated average wind flow pressure in the "virtual drainage point " at the facade of the building in averaging several temporary quasi-period fluctuations; $\rho$ is an air density; $U(H)$ is depending on the height $\mathrm{H}$ the average flow velocity in the wind lay.

Based on the pattern of distribution of the average coefficient of aerodynamic pressure on the facades it is impracticable to determine the dimensionless coefficient $C p$, that will be applied in the calculation of wind loads on the building of the airport complex.

Fig. 7 is a picture of the distribution of minima and maxima peak on the facades of the airport terminal complex. 


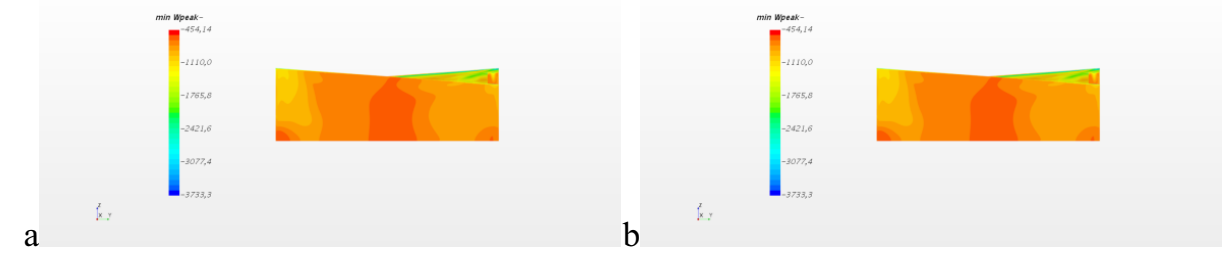

Fig. 7. Distribution minima (a) and highs (b) peak (Wpeak- and Wpeak + ) on the facades of the airport terminal complex.

\section{Conclusions}

Simulation of flow of the airport terminal using provided in this article computer technology shows information on the distribution of the wind effects on the building facades. The data medium aerodynamic coefficient in computational research provide a good consistency with the experimental results and allow us to determine the design wind loads on the facade of the airport complex in accordance with the SP 20.13330.2011 "Loads and effects". The accepted method of determining the coefficient Cp corresponds to the requirements of normative documents on the implementation of such works.

This work was financially supported by the Council for Grants of the President of the Russian Federation for state support of young Russian scientists in the framework of the research MK6194.2015.8.

\section{References}

1. Building codes SP 20.13330.2011 Loads and effects. The updated edition of SNIP 2.01.07-85 (2011)

2. A. Kubenin, A. Fedosova, O. Poddaeva, Scientific Review 21, 368-372 (2015)

3. A. Kubenin, A. Fedosova, The National Association of Scholars X(15) 99-102 (2015)

4. Al Zoubi Feras, Li Zheng-liang, Wei Qi-ke, Sun Yi, Journal of Chongqing University 1(9) $47-53$ (2010)

5. The use of Wind tunnel experiments for wind loads on structures. C.P.W. (Geurts TNO Built Environment and Geosciences, Delft)

6. Wind Tunnel Testing of High-Rise Buildings (An output of the CTBUH Wind Engineering Working Group, 2013).

7. O.O. Egorychev, S.I. Dubinsky, A.N. Fedosova, Applied Mechanics and Materials 713-715, 1729 (2015)

8. O.I. Poddaeva, S.I. Dubinsky, A. Fedosova, Industrial and civil engineering 9, 23-27 (2014)

9. V. Gagarin, S.V. Guvernyuk, A.S. Kubenin, Housing construction 7, 3-8 (2014) 